\title{
LONGITUDINAL SHOCK SOLID OBJECT ONTO THE ELASTIC BAR PLACED ON THE VISCO-ELASTIC FOUNDATION WITH CONSTANT RESISTANCE OF A PART OF THE BAR SIDE FACE
}

\section{NGUYeN ThuC AN, NguYen ThI ThanH BINH Hanoi Water Resources University}

\section{Introduction}

Basing on the theory of one-dimensional wave, authors have studied the problem of longitudinal shock of solid object onto the elastic bar placed on the viscoelastic foundation with constant resistance of a part of the bar side face.

The purpose of this paper is to determine stress state of the bar. The diagram of the problem is
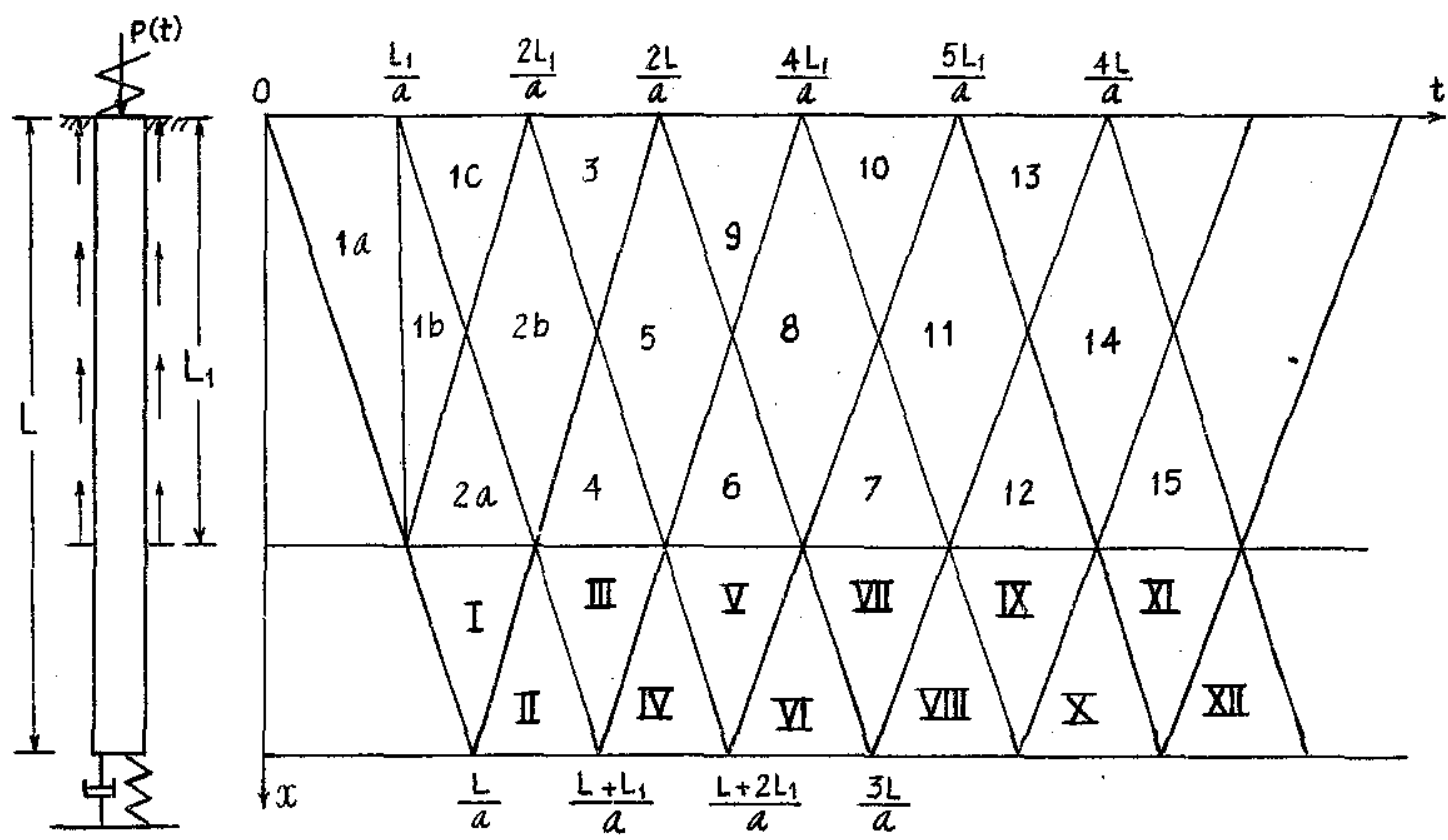

2. Formulation of the problem

2.1. The equation of motion of the part of the side face of the bar with 
constant resistance is determined as

$$
\frac{\partial^{2} U}{\partial t^{2}}=a^{2}\left(\frac{\partial^{2} U}{\partial x^{2}}-K_{1}\right) \quad \text { with } \quad t>0 ; \quad 0 \leq x \leq L_{1}
$$

The general solution of Eq.(2.1) bounded on the zone $1 \mathrm{a}$ is

$$
U_{1}(x, t)=\varphi_{1}(a t-x)+\frac{1}{2} K_{1} x^{2}-K_{1} a t x
$$

The general solution of Eq.(2.1) on the zones $1 \mathrm{~b}, 1 \mathrm{c}$ is

$$
U_{1}(x, t)=\varphi_{1}(a t-x)+\frac{1}{2} K_{1}\left(L_{1}-x\right)^{2} .
$$

The general solution of Eq.(2.1) on the different zones is

$$
U_{1}(x, t)=\varphi_{1}(a t-x)+\psi_{1}(a t+x)+\frac{1}{2} K_{1}\left(L_{1}-x\right)^{2} .
$$

2.2. The equation of motion of the other part of the bar is

$$
\frac{\partial^{2} U_{2}}{\partial t^{2}}=a^{2} \frac{\partial^{2} U_{2}}{\partial x^{2}} \quad \text { with } \quad L_{1}<x \leq L ; t \geq \frac{L_{1}}{a} .
$$

Therefore we have

$$
U_{2}(x, t)=\varphi_{2}(a t-x)+\psi_{2}(a t+x)
$$

Initial condition:

At $t=0$

$$
\begin{array}{ll}
U_{1}=0 ; \quad \frac{\partial U_{1}}{\partial x}=0 ; \quad \frac{\partial U_{1}}{\partial t}=0 \quad \text { with } 0 \leq x \leq L_{1} . \\
U_{2}=0 ; \quad \frac{\partial U_{2}}{\partial x}=0 ; \quad \frac{\partial U_{2}}{\partial t}=0 \quad \text { with } L_{1} \leq x \leq L .
\end{array}
$$

Boundary condition:

At the shocked end of the bar $x=0$

$$
\frac{\partial U_{1}}{\partial x}=-\frac{P(t)}{E F} .
$$

At $x=L_{1}$

$$
\frac{\partial U_{1}}{\partial x}=\frac{\partial U_{2}}{\partial x} ; \quad \frac{\partial U_{1}}{\partial t}=\frac{\partial U_{2}}{\partial t}
$$


At $x=L$

$$
\frac{\partial U_{2}}{\partial x}=-K_{2} U_{2}-\lambda \frac{\partial U_{2}}{\partial t}
$$

where $K_{2}$ and $\lambda$ are elastic and viscid coefficients respectively. They are considered is constants.

\section{Determination of the wave functions of the bar}

3.1. Considering wave functions in a limited duration $0 \leq t \leq \frac{L_{1}}{a}$

According to [3] the force of the cap on the shocked head of the bar is

$$
P_{0}(t)=e^{-n t}\left(C_{1} \cos \omega_{1} t+C_{2} \sin \omega_{1} t\right)+\frac{C a^{2} K_{1}}{\omega_{1}^{2}+n^{2}},
$$

where

$$
C_{1}=-\frac{C a^{2} K_{1}}{\omega_{1}^{2}+n^{2}} ; \quad C_{2}=\frac{1}{\omega_{1}}\left(C V-\frac{C a^{2} K_{1} n}{\omega_{1}^{2}+n^{2}}\right) .
$$

Basing on (2.6a) and (2.2a) the wave function $\varphi_{1}^{\prime}(a t-x)$ on the domain 1a is determined as

$$
\varphi_{1}^{\prime}(a t-x)=\frac{1}{E F} P_{0}\left(t-\frac{x}{a}\right)-K_{1}(a t-x) .
$$

According to the condition at the boundary of the zones $1 \mathrm{a}$ and $1 \mathrm{~b}$ with $t=L_{1} / \mathrm{a}$ the function $U_{1}(x, t)$ is a continuous function on the regions $1 \mathrm{~b}, 2 \mathrm{a}$ and $\varphi_{1}^{\prime}(a t-x)$ has the form (3.2).

From condition $(2.6 \mathrm{~b})$ we have

$$
\psi_{2 a}^{\prime}\left(a t+L_{1}\right)=\psi_{1}^{\prime}\left(a t+L_{1}\right) ; \quad \varphi_{2 a}^{\prime}\left(a t-L_{1}\right)=\varphi_{1}^{\prime}\left(a t-L_{1}\right) .
$$

With the limited duration $0 \leq t \leq \frac{L_{1}}{a}$ the wave function $\psi_{1}^{\prime}\left(a t+L_{1}\right)=0$, we have $\psi_{2 a}\left(a t+L_{1}\right)=0$, so that $\psi_{2 a}^{\prime}(a t+x)=0$.

Basing on (3.2) we have $\varphi_{2}^{\prime}(a t-x)$ of the bar in the zonez I, II:

$$
\varphi_{2}^{\prime}(a t-x)=\frac{1}{E F} P_{0}\left(t-\frac{x}{a}\right)-K_{1}(a t-x) .
$$

On the zones $1 \mathrm{a}, 1 \mathrm{~b}, 2 \mathrm{a}$ and 3 we have $\psi_{1}^{\prime}(a t+x)=0, \psi_{2}^{\prime}(a t+x)=0$.

According to $(2.6 \mathrm{c})$ we have

$$
\psi_{2}^{\prime}(Z)+\frac{K_{2}}{1+a \lambda} \psi_{2}(Z)=\frac{1-a \lambda}{1+a \lambda} \varphi_{2}^{\prime}(Z-2 L)-\frac{K_{2}}{1+a \lambda} \varphi_{2}(Z-2 L),
$$


with $Z=a t+L$.

The general solution of Eq.(3.5), is

$$
\psi_{2}(Z)=H e^{\frac{-K_{2} Z}{1+a \lambda}}+e^{\frac{-K_{2}(z-2 L)}{1+a \lambda}} \int e^{\frac{K_{2}}{1+a \lambda} \tau}\left[\frac{1-a \lambda}{1+a \lambda} \varphi_{I I}^{\prime}(\tau)-\frac{K_{2}}{1+a \lambda} \varphi_{I I}(\tau)\right] d \tau
$$

Basing on the continuous characteristics of function $U_{2}(x, t)$ at the time $t=\frac{L}{a}$ and $x=L$, we have: $\psi_{2}\left(L, \frac{L}{a}\right)=0$ and $H=H_{0}=0$, where $H_{0}$ is integral constant of $H$ in the limited duration $\frac{L}{a} \leq t \leq \frac{L+L_{1}}{a}$. So that

$$
\psi_{2}(Z)=e^{-\frac{K_{2}}{1+a \lambda}(Z-2 L)} \int_{0}^{Z-2 L} e^{\frac{K_{2}}{1+a \lambda} \tau}\left[\frac{1-a \lambda}{1+a \lambda} \varphi_{I I}^{\prime}(\tau)-\frac{K_{2}}{1+a \lambda} \varphi_{I I}(\tau)\right] d \tau
$$

The wave function $\psi_{2}^{\prime}(Z)$ at the bar end with $\frac{L}{a} \leq t \leq \frac{L+L_{1}}{a}$ is

$$
\begin{aligned}
\psi_{2}^{\prime}(Z)= & -\frac{K_{2}}{1+a \lambda} e^{\frac{K_{2}}{1+a \lambda}(Z-2 L)} \int_{0}^{Z-2 L} e^{\frac{K_{2}}{1+a \lambda}} \tau\left[\frac{1-a \lambda}{1+a \lambda} \varphi_{I I}^{\prime}(\tau)-\frac{K_{2}}{1+a \lambda} \varphi_{I I}(\tau)\right] d \tau \\
& +\frac{1-a \lambda}{1+a \lambda} \varphi_{I I}^{\prime}(Z-2 L)-\frac{K_{2}}{1+a \lambda} \varphi_{I I}(Z-2 L) .
\end{aligned}
$$

The wave function $\psi_{2}^{\prime}(a t+x)$ on the zones II and III in the bar is

$$
\begin{aligned}
& \psi_{2}^{\prime}(a t+x)= \\
& =-\frac{K_{2}}{1+a \lambda} e^{-\frac{K_{2}}{1+a \lambda}(a t+x-2 L)} \int_{0}^{a t+x-2 L} e^{\frac{K_{2}}{1+a \lambda} \tau}\left[\frac{1-a \lambda}{1+a \lambda} \varphi_{I I}^{\prime}(\tau)-\frac{K_{2}}{1+a \lambda} \varphi_{I I}(\tau)\right] d \tau \\
& \quad+\frac{1-a \lambda}{1+a \lambda}\left[\varphi_{I I}^{\prime}(a t+x-2 L)-\frac{K_{2}}{1+a \lambda} \varphi_{I I}(a t+x-2 L)\right]
\end{aligned}
$$

where

$$
\begin{aligned}
& \varphi_{I I}^{\prime}(\tau)= \frac{1}{E F a} P_{0}(\tau)-K_{1} \tau=\frac{1}{E F a}\left[e^{-n \tau}\left(C_{1} \cos \omega_{1} \tau+C_{2} \sin \omega_{1} \tau\right)+\frac{C a^{2} K_{1}}{\omega_{1}^{2}+n^{2}}\right]-K_{1} \tau \\
& \varphi_{I I}(\tau)=\int_{0}^{1} \varphi_{I I}^{\prime}(\tau) d \tau= \\
&=\frac{e^{-n \tau}}{E F a\left(\omega_{1}^{2}+n^{2}\right)}\left[\left(C_{1} \omega_{1}-C_{2} n\right) \sin \omega_{1} \tau-\left(C_{1} n+C_{2} \omega_{1}\right) \cos \omega_{1} \tau\right] \\
&-\frac{K_{1}}{2} \tau^{2}+\frac{C a^{2} K_{1} \tau}{E F a\left(\omega_{1}^{2}+n^{2}\right)}+\frac{C_{1} n+C_{2} \omega_{1}}{E F a\left(\omega_{1}^{2}+n^{2}\right)}
\end{aligned}
$$


According to condition (3.3) the wave function $\psi_{1}^{\prime}(a t+x)$ on the zones 4,5 and 9 in the bar also has the form (3.7).

3.2. Considering wave functions in a limited duration $\frac{L_{1}}{a} \leq t \leq \frac{2 L}{a}$ with $L=L_{1}+\frac{L_{1}}{2}$

From [3] impact-pressing force of the cap on the shocked head of the bar with this limited time has the form

$$
P_{1}(t)=e^{-n t}\left(C_{3} \cos \omega_{1} t+C_{4} \sin \omega_{1} t\right)
$$

where

$$
\begin{aligned}
& C_{3}=e^{\frac{n L_{1}}{a}} P_{0}\left(\frac{L_{1}}{a}\right) \cos \frac{\omega_{1} L_{1}}{a}-\frac{1}{\omega_{1}} e^{\frac{n L_{1}}{a}}\left[n P_{0}\left(\frac{L_{1}}{a}\right)+\dot{P}_{0}\left(\frac{L_{1}}{a}\right)\right] \sin \omega_{1} L_{1}, \\
& C_{4}=\frac{1}{\omega_{1}} e^{\frac{n L_{1}}{a}}\left[n P_{1}\left(\frac{L_{1}}{a}\right)+\dot{P}_{0}\left(\frac{L_{1}}{a}\right)\right] \cos \frac{\omega_{1} L_{1}}{a}+e^{\frac{n L_{1}}{a}} \dot{P}_{0}\left(\frac{L_{1}}{a}\right) \sin \omega_{1} L_{1} .
\end{aligned}
$$

According to conditions (2.6b) and (2.2b) the wave function $\varphi_{1}^{\prime}(a t-x)$ on the zones $1 \mathrm{c}, 2 \mathrm{~b}, 4,5$ and 6 in the bar is

$$
\varphi_{1}^{\prime}(a t-x)=\frac{1}{E F} P_{1}\left(t-\frac{x}{a}\right)-K_{1} L_{1}
$$

Basing on (2.6b) the wave function $\varphi_{2}^{\prime}(a t-x)$ on the zones III, IV, V and VI also has the form (3.8).

From condition (2.6c) we have

$$
\psi_{2}^{\prime}(Z)+\frac{K_{2}}{1+a \lambda} \psi_{2}(Z)=\frac{1-a \lambda}{1+a \lambda} \varphi_{2}^{\prime}(Z-2 L)-\frac{K_{2}}{1+a \lambda} \varphi_{2}(Z-2 L)
$$

The general solution of this equation is

$$
\begin{aligned}
\psi_{2}(Z)= & H_{1} e^{-\frac{K_{2}}{1+a \lambda} Z} \\
& +e^{-\frac{K_{2}}{1+a \lambda}(Z-2 L)} \int_{L_{1}}^{\left(Z_{-2 L)}\right.} e^{\frac{K_{2}}{1+a \lambda} \tau}\left[\frac{1-a \lambda}{1+a \lambda} \varphi_{I V}^{\prime}(\tau)-\frac{K_{2}}{1+a \lambda} \varphi_{I V}(\tau)\right] d \tau
\end{aligned}
$$

where $H_{1}$ is integral constant, which is determined from continuous characteristic of the function $U_{2}(t, x)$ at $x=L_{;} t=\frac{L+L_{1}}{a}$. 
Basing on conditions (3.6a) and (3.6b) we have

$$
e^{-\frac{K_{2}}{1+a \lambda} L_{1}} \int_{0}^{L_{1}} e^{\frac{K_{2} \tau}{1+a \lambda}}\left[\frac{1-a \lambda}{1+a \lambda} \varphi_{I I}^{\prime}(\tau)-\frac{K_{2}}{1+a \lambda} \varphi_{I I}(\tau)\right] d \tau=H_{1} e^{-\frac{K_{2}}{1+a \lambda}\left(2 L+L_{1}\right)}
$$

So that

$$
H_{1}=e^{\frac{K_{2} \cdot 2 L}{1+a \lambda}} \int_{0}^{L_{1}} e^{\frac{K_{2} \tau}{1+a \lambda}}\left[\frac{1-a \lambda}{1+a \lambda} \varphi_{I I}^{\prime}(\tau)-\frac{K_{2}}{1+a \lambda} \varphi_{I I}(\tau)\right] d \tau
$$

The wave function $\psi_{2}(a t+x)$ on zones IV, V, VI and VII in the bar is

$$
\begin{aligned}
& \psi_{2}(a t+x)=H_{1} e^{-\frac{K_{2}}{1+a \lambda}(a t+x)} \\
& +e^{-\frac{K_{2}}{1+a \lambda}(a t+x-2 L)} \int_{L_{1}}^{a t+x-2 L} e^{\frac{K_{2} r}{1+a \lambda}}\left[\frac{1-a \lambda}{1+a \lambda} \varphi_{I V}^{\prime}(\tau)-\frac{K_{2}}{1+a \lambda} \varphi_{I V}(\tau)\right] d \tau .
\end{aligned}
$$

So, the wave function $\psi_{2}^{\prime}(a t+x)$ on the zones IV, V, VI and VII in the bar is

$$
\begin{aligned}
\psi_{2}^{\prime}(a t+x)= & -\frac{K_{2}}{1+a \lambda}\left\{H_{1} e^{-\frac{K_{2}}{1+a \lambda}(a t+x)}+e^{-\frac{K_{2}}{1+a \lambda}(a t+x-2 L)}\right. \\
& \left.\int_{L_{1}}^{a t+x-2 L} e^{\frac{K_{2}}{1+a \lambda} \tau}\left[\frac{1-a \lambda}{1+a \lambda} \varphi_{I V}^{\prime}(\tau)-\frac{K_{2}}{1+a \lambda} \varphi_{I V}(\tau)\right] d \tau\right\} \\
& +\frac{1-a \lambda}{1+a \lambda} \varphi_{I V}^{\prime}(a t+x-2 L)-\frac{K_{2}}{1+a \lambda} \varphi_{I V}(a t+x-2 L)
\end{aligned}
$$

where

$$
\begin{aligned}
\varphi_{I V}^{\prime}(\tau)= & \frac{1}{E F a} P_{1}(\tau)=\frac{1}{E F a} e^{-n t}\left(C_{3} \cos \omega_{1} \tau+C_{4} \sin \omega_{1} \tau\right)-K_{1} L \\
\varphi_{I V}(\tau)= & \int_{L_{1}}^{\tau} \varphi_{I V}^{\prime}(\tau) d \tau=-\frac{C_{4} \omega_{1}+C_{3} n}{E F a\left(\omega_{1}^{2}+n^{2}\right)} e^{-n \tau} \cdot \cos \omega_{1} \tau \\
& +\frac{C_{3} \omega_{1}-C_{4} n}{E F a\left(\omega_{1}^{2}+n^{2}\right)} e^{-n t} \sin \omega_{1} \tau-K_{1} L_{1} \tau+F
\end{aligned}
$$

with

$$
F=\frac{C_{4} \omega_{1}+C_{3} n}{E F a\left(\omega_{1}^{2}+n^{2}\right)} e^{-n L_{1}} \cos \omega_{1} L_{1}+\frac{C_{4} n-C_{3} \omega_{1}}{E F a\left(\omega_{1}^{2}+n^{2}\right)} e^{-n L_{1}} \sin \omega_{1} L_{1}
$$


From condition (3.3) on zones $6,7,8,10,11$ and 13 in the bar the wave function $\psi_{1}^{\prime}(a t+x)$ also has form (3.9). If the shock of a solid object to bar is still not finished yet in section $0<t<\frac{2 L}{a}$, the study of the problem in the next sections is similar to the above section.

\section{Determination of stress status of the bar}

We have in zore $1 \mathrm{a}$

$$
\sigma=E\left[-\varphi_{1}^{\prime}(a t-x)+K_{1}(x-a t)\right],
$$

in zones $1 b, 2 a, 2 b$ and 3

$$
\sigma=E\left[-\varphi_{1}^{\prime}(a t-x)+K_{1}\left(L_{1}-x\right)\right]
$$

in the remained zones

$$
\sigma=E\left[-\varphi_{1}^{\prime}(a t-x)+\psi_{1}^{\prime}(a t+x)-K_{1}\left(L_{1}-x\right)\right] .
$$

The stress on the zones in the bar without resistance has the form

$$
\sigma=E\left[-\varphi_{2}^{\prime}(a t-x)+\psi_{2}^{\prime}(a t+x)\right] .
$$

From conditions (4.1) and (3.2) we have the stress on zone 1a in the bar:

$$
\sigma=-\frac{1}{F} P_{0}\left(t-\frac{x}{a}\right) \text {. }
$$

Basing on (4.2) and (3.2) the stress on zones $1 \mathrm{~b}$ and $2 \mathrm{a}$ in the bar has the form

$$
\sigma=-\frac{1}{F} P_{1}\left(t-\frac{x}{a}\right)+E K_{1}\left(a t-L_{1}\right) .
$$

According to the formula (4.4) and (3.4) we have the stress on zone $I$ in the bar

$$
\sigma=-\frac{1}{F} P_{0}\left(t-\frac{x}{a}\right)+E K_{1}\left(a t-L_{1}\right) \text {. }
$$

Basing on (4.4), (3.4) and (3.7) the stress on zone II in the bar has form

$$
\begin{aligned}
\sigma= & E\left\{-\frac{1}{E F} P_{0}\left(t-\frac{x}{a}\right)+K_{1}(a t-x)-\frac{K_{2}}{1+a \lambda} e^{-\frac{K_{2}}{1+a \lambda}(a t+x-2 L)} .\right. \\
& \int_{0}^{a t+x-2 L} e^{\frac{K_{2}}{1+a \lambda} \tau}\left[\frac{1-a \lambda}{1+a \lambda} \varphi_{I I}^{\prime}(\tau)-\frac{K_{2}}{1+a \lambda} \varphi_{I I}(\tau)\right] d \tau+ \\
& \left.+\frac{1-a \lambda}{1+a \lambda} \varphi_{I I}^{\prime}(a t+x-2 L)-\frac{K_{2}}{1+a \lambda} \varphi_{I I}(a t+x-2 L)\right\}
\end{aligned}
$$


From (3.8) and (4.2) the stress on zones $1 c, 2 b$ and 3 in the bar is

$$
\sigma=\frac{P_{1}\left(t-\frac{x}{a}\right)}{F}+E K_{1} L_{1} .
$$

According to (3.7), (3.8) and (4.3) the stress on zones 4 and 5 in the bar has the form

$$
\begin{aligned}
\sigma= & E\left\{-\frac{1}{E F} P_{1}\left(t-\frac{x}{a}\right)-K_{2} e^{-K_{2}(a t+x-2 L)} .\right. \\
& \int_{0}^{a t+x-2 L} e^{K_{2} \tau}\left[\frac{1-a \lambda}{1+a \lambda} \varphi_{I I}^{\prime}(\tau)-\frac{K_{2}}{1+a \lambda} \varphi_{I I}(\tau)\right] d \tau \\
& \left.+\frac{1-a \lambda}{1+a \lambda} \varphi_{I I}^{\prime}(a t+x-2 L)-\frac{K_{2}}{1+a \lambda} \varphi_{I I}(a t+x-2 L)+K_{1} x\right\} .
\end{aligned}
$$

From (3.7), (3.8) and (4.4) the stress on zone III in the bar is

$$
\begin{aligned}
\sigma= & E\left\{-\frac{1}{E F} P_{1}\left(t-\frac{x}{a}\right)+K_{1} L_{1}-\frac{K_{2}}{1+a \lambda} e^{-\frac{K_{2}}{1+a \lambda}(a t+x-2 L)} .\right. \\
& \int_{0}^{a t+x-2 L} e^{\frac{K_{2}}{1+a \lambda} \tau}\left[\frac{1-a \lambda}{1+a \lambda} \varphi_{I I}^{\prime}(\tau)-\frac{K_{2}}{1+a \lambda} \varphi_{I I}(\tau)\right] d \tau \\
& \left.+\frac{1-a \lambda}{1+a \lambda} \varphi_{I I}^{\prime}(a t+x-2 L)-\frac{K_{2}}{1+a \lambda} \varphi_{I I}(a t+x-2 L)\right\}
\end{aligned}
$$

Basing on (4.4), (3.9) and (3.8) the stress on zones IV, V, VI in the bar will be

$$
\begin{aligned}
\sigma= & E\left\{-\frac{1}{E F} P_{1}\left(t-\frac{x}{a}\right)+K_{1} L_{1}-\frac{K_{2}}{1+a \lambda}\left\{H_{1} e^{-\frac{K_{2}}{1+a \lambda}(a t+x)}+e^{-\frac{K_{2}}{1+a \lambda}(a t+x-2 L)}\right.\right. \\
& \left.+\int_{L_{1}}^{a t+x-2 L} e^{\frac{K_{2}}{1+a \lambda} \tau} \cdot\left[\frac{1-a \lambda}{1+a \lambda} \varphi_{I V}^{\prime}(\tau)-\frac{K_{2}}{1+a \lambda} \varphi_{I V}(\tau)\right] d \tau\right\} \\
& \left.+\frac{1-a \lambda}{1+a \lambda} \varphi_{I V}^{\prime}(a t+x-2 L)-\frac{K_{2}}{1+a \lambda} \varphi_{I V}(a t+x-2 L)\right\} .
\end{aligned}
$$

From (4.3), (3.9) and (3.8) the stress on zone 6 in the bar has the form:

$$
\begin{aligned}
\sigma= & E\left\{-\frac{1}{E F} P_{1}\left(t-\frac{x}{a}\right)-\frac{K_{2}}{1+a \lambda}\left\{H_{1} e^{-\frac{K_{2}}{1+a \lambda}(a t+x)}+e^{-\frac{K_{2}}{1+a \lambda}(a t+x-2 L) .}\right.\right. \\
& \left.\int_{L_{1}}^{a t+x-2 L} e^{\frac{K_{2}}{1+a \lambda} \tau}\left[\frac{1-a \lambda}{1+a \lambda} \varphi_{I V}^{\prime}(\tau)-\frac{K_{2}}{1+a \lambda} \varphi_{I V}(\tau)\right] d \tau\right\} \\
& \left.+\frac{1-a \lambda}{1+a \lambda} \varphi_{I V}^{\prime}(a t+x-2 L)-\frac{K_{2}}{1+a \lambda} \varphi_{I V}(a t+x-2 L)+K x\right\} .
\end{aligned}
$$


By the same way we can determine the stress on the remaining zones in the bar.

\section{Conclusion}

Using the well known wave method the authors study the problem of longitudinal shock of solid object on the elastic bar placed on the visco-elastic foundation with constant resistance of a part of the bar side face. The purpose of this paper is to determine stress state of the bar.

Mechanically, boundary restrains in this problem are wider than some problems studied in [1-3].

Technically, this model is similar to the problem of piling in a non-homogeneous foundation.

The research is partly supported by the council for Natural Sciences of Vietnam.

\section{References}

1. Gecsevanow N. M. Theory of longitudinal shock of solid object on the elastic bar and application of earth resistance. Bulletin on the foundation mechanics No. 1 Moscow 1948.

2. Nguyen Thi Thanh Binh. Longitudinal shock of solid body or the elastic bar which is based one the visco-elastic foundation with constant resistant of the side faces. Vietnam Journal of Science and Technology No. 5, 1997.

3. Nguyen Thuc An, Nguyen Viet Quang, Nguyen Thi Thanh Binh, Pham Hong Anh. Shock of hammer onto pile on the solid foundation. Selection of Scientific Research works of Hanoi Water Resources University 8-1996.

Received February 15, 1998

VA CHAM CỨA VẬT RẮN VÀO THANH ĐÀN HỒI TỰA TRÊN

NỀN ĐÀN NHỚT MỘT PHẦN MẶT BÊN CHỊU LỰC CẢN KHÔNG ĐỔI

Trên cơ sớ lý thuyết sóng một chiều với nghiệm $\mathrm{Da}$ lăm be các tác giả đã xét bài toán va chạn của vật rắn vào thanh đàn hồi qua đệm giảm chấn tuyến tính, một phần của thanh chịu lực cản không đổi và đầu kia của thanh chịu lực cán đàn nhớt.

Nội dung chính là tìm các hàm sóng $\varphi^{\prime}(a t-x)$ và $\psi^{\prime}(a t+x)$ và xác định trạng thái ứng suất cưa thanh trong thời gian va chạm. 